\title{
Using LQI to Improve ClusterHead Locations in Dense ZigBee based Wireless Sensor Networks
}

\author{
Chérif Diallo, Michel Marot, Monique Becker \\ SAMOVAR CNRS Research Lab - UMR 5157; Dept Réseaux et Services de Télécommunications (RST) \\ Institut TELECOM; TELECOM SudParis; 9, Rue Charles Fourier - 91011 Evry CEDEX, France \\ Email:\{cherif.diallo, michel.marot, monique.becker\}@ telecom-sudparis.eu
}

\begin{abstract}
In WSN, it is not often desirable to use the GPS technology. Indeed, the use of GPS is expensive and may reduce the overall network performance. Moreover, indoor reception of the GPS signal is not possible. The Link Quality Indicator (LQI) is defined in the 802.15.4 standard, but its context of use is not specified in this standard. Some works on the LQI, few of which are field experiments, have shown that the LQI decreases as the distance increases. However, the challenge of clustering mechanisms is to form the smallest number of clusters by maximizing distances separating cluster heads to provide an efficient cover of the network and also minimizes the cluster overlaps. This reduces the amount of channel contention between clusters, and also improves the efficiency of algorithms that run at the level of the cluster heads. Therefore we propose an analytical model based on the use of the LQI in order to derive an optimally one-dominating set in which the smaller distance separating two cluster heads is improved.

Index Terms-WSN Clusters; LQI; Clusterhead locations.
\end{abstract}

\section{INTRODUCTION}

In the Zigbee standard [1],[2], the LQI measurement is defined as a characterization of the strength and/or quality reception of a packet. The use of the LQI result by the network or the application layers is not specified in [1],[2]. The LQI measurement is performed for each received packet, and the result is reported to the MAC sublayer as an integer ranging from 0 to 255 . The minimum and maximum LQI values (0 and 255) are associated with the lowest and the highest quality IEEE 802.15.4 reception detectable by the receiver, and the LQI values in between are distributed between these two limits [1],[2].

In this paper, we propose an analytical model based on the use of the LQI in order to derive an heuristic which forms an optimally one-dominating set in which the distance separating the two closest cluster heads is improved. During the clustering process, each caryomme selects in its 1-hop neighbourhood some nodes which are its emissaries. The relationship between two adjacent clusters is then created by the best emissary node located between the two respective cluster heads. Consequently, at the end of the clustering process, the set composed of the cluster heads and the best emissary nodes forms a connected backbone in terms of data routing.
In an homogeneous WSN where all nodes have the same transmission power, the LQI essentially depends on the distance separating the nodes. The caryommes and their respective emissary nodes use the quality of the links they form with its respective neighbours. The LQI is used in order to take into account the distance separating nodes. As the GPS technology is expensive and impossible to use in an indoor environment, it is not often desirable to add GPS futures in the WSN. Furthermore, in some applications, it is not a requirement to accurately determine the node locations. In such applications it is only sufficient to have an idea on the remoteness of some nodes with respect to any given sensor.

The rest of this paper is organized as follows. After an overview of the related works in the next part, the next one gives an understanding formulation of the problem. In the fourth part, we present the analytical model and then derive a global heuristic. The last section presents simulation results.

\section{State of THE ART}

In Zigbee [1],[2] a cluster-tree topology is provided with the standard 802.15.4 std. This cluster-tree topology is well suited to heterogeneous networks but is not fully scalable for large homogeneous WSN. Few papers concern LQI based clustering protocols for WSN. In [3], authors propose a link-state clustering algorithm (LSCA) for both homogeneous and heterogeneous large scale wireless sensor networks. The LEACH [4] algorithm for selecting clusterheads is a probabilistic method which produces clusters with a large variation of link distances and uneven energy consumption during the data transmission phase. To address this issue, a distributed algorithm is presented in [5] in which nodes attempt to form favourable clusters based on Received Signal Strength indication (RSSI) density and quality. These papers do not take into account multihop clustering aspects. Multihop clustering algorithms address two main challenges: first, the question arises how to optimally choose caryommes with respect to their locations and, secondly, how to construct the parental relationship between regular nodes and their caryommes in such a way that any common sensor can reach its clusterhead within k hops, that is to say, how to construct an optimally independent k-dominating set. Unfortunately, finding such a set is an NP-complete problem [6], so some 
heuristics have been proposed [7],[8],[9]. In [10] authors proposed three algorithms to build k-dominating sets which are also k-connected.

In [11], authors proposed a Self Organizing Sensor (SOS) network based on an intelligent clustering algorithm which is aimed to reduce channel contention and cluster overlaps. This paper does not focus on the use of LQI. It does not also present results on clusterhead locations.

Although all these studies provide a valuable and strong contribution in WSN clustering, the problem of optimizing the distance between the two closest cluster heads is now to addressed. We then present an analytical model based on the use of the LQI as the usability of the GPS technology is not possible in an indoor environment.

\section{PRoblem Formulation}

The WSN can be modelled as a graph $G=(V, E)$, where two nodes are connected by an edge if they can communicate with each other. Let $x \in V$ be a node in the WSN. $\mathcal{N}_{1}(x)$ is the neighbourhood of the node $x$. A node $x$ forms with each $y \in \mathcal{N}_{1}(x)$ a link of which the link quality indicator LQI value is denoted by $\ell(x, y)>0$. For all other nodes $z \in V \backslash \mathcal{N}_{1}(x)$, $\ell(x, z)=0$. Let $\nu$ be a bijective function defined in $V$ which is a totally ordered set. The $\nu$ function is defined as follows:

$$
\forall x \in V, \nu(x)=(f(x), i d(x))
$$

where $f(x)$ is the criteria function and $i d(x)$ returns the address of the node $x$. The total ordering in $V$ is defined as follows:

$$
\begin{aligned}
& \forall x \in V, \nu(x)>\nu(y) \Longleftrightarrow(f(x)>f(y)) \\
& \text { or } \quad(f(x)=f(y) \quad \text { and } \quad i d(x)>i d(y))
\end{aligned}
$$

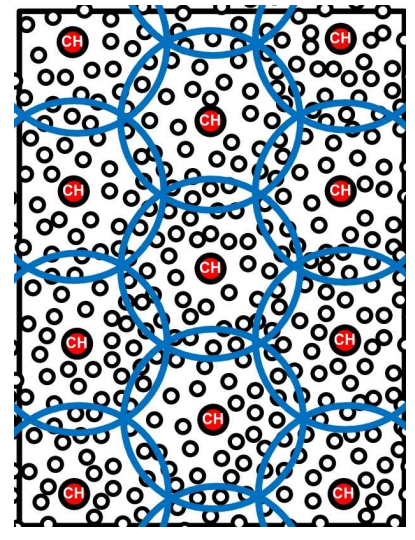

(a) WSN Clustering

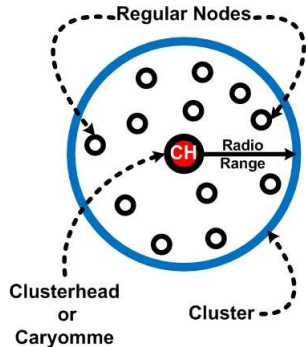

(b) Cluster
Fig. 1. WSN Clustering

The clustering problem intended to construct an optimally $\mathrm{k}$-dominating set $(k=1)$ can be defined as follows. Assume that nodes are randomly distributed in a field. Each cluster consists of a single cluster head and some regular nodes as illustrated in figures 1(a) and 1(b). At the end of the clustering process, each regular node belongs (at least) to one cluster and is able to directly communicate with its cluster head which is located in its 1-hop neighbourhood. The challenge of clustering mechanisms is to form the smallest number of clusters by maximizing distances separating cluster heads in such a way that each regular node is at most at one wireless hop away from its cluster head. Maximizing the distances separating the cluster heads provides an efficient cover of the whole network and also minimizes the cluster overlaps. This reduces the amount of channel contention between clusters, and also improves the efficiency of algorithms that run at the level of the cluster heads.

At the end of the clustering process, if we denote by $\mathcal{S}$ the set of elected cluster heads, then the set $\Omega$ of the clusterized nodes defined by :

$$
\Omega=\left\{x \in V, \quad \exists y \in \mathcal{S} \cap \mathcal{N}_{1}(x)\right\}
$$

Hence, to maximize the distances separating the cluster heads, we have to maximize the distance separating the two cluster heads which are the closest cluster heads in the whole network. Let $d(x, y)$ the function which returns the euclidian distance separating two clusterheads $(x, y) \in \mathcal{S}$. The problem consists then in maximizing the following function:

$$
\left(\mathcal{P}_{b}\right)=\max _{\mathcal{S}} \min _{(x, y) \in \mathcal{S}} d(x, y)
$$

under the two constraints $\left(\mathcal{C}_{1}\right)$ and $\left(\mathcal{C}_{2}\right)$ :

- $\left(\mathcal{C}_{1}\right): \mathcal{S}$ forms a 1-dominant subset of $V$.

- $\left(\mathcal{C}_{2}\right)$ : The area $\bar{\Omega}$ formed by the zone bounded by the peripheral nodes of $\Omega$ is convex.

The constraint $\left(\mathcal{C}_{2}\right)$ means that there should not be any non clusterized node inside the area bounded by different clusters.

\section{PAPER ObJectives}

The LQI is defined in the 802.15.4 standard [1],[2], but its context of use is not yet specified in the standard. Therefore we propose the use of the link quality for the cluster formation in WSN. The main idea is based on the fact that the LQI gives an idea of the distance separating the two nodes that form the link. Indeed, the LQI decreases as the distance increases [12],[13]. In an homogeneous WSN where all sensors have the same transmission power, the LQI only depends on the distance. To avoid using GPS technology which is expensive and is also impossible to use in an indoor environment, it is useful to exploit the LQI that is already defined at the MAC sublayer of Zigbee based WSNs. The second objective is to propose a cluster formation where clusterheads are sufficiently outspread from each other. In [14], we have improved the MaxMin [6] d-cluster formation in WSN by reducing the single node clusters and then compared energy efficiency of several criteria in MaxMin. This work [14] has shown that criteria that minimize the cluster overlaps help in reducing 
the amount of channel contention between clusters, and also improve the overall energy efficiency of the network.

This paper presents a model to improve clustering in dense wireless sensor networks by using LQI as an indication of distance. This topic is of great importance, and the use of LQI to control the distance of cluster heads could be new. However, the problem is simplified in this paper, and some issues are not discussed. The degradation in channel quality, the mobility and the dynamic in wireless environment are not considered.

The paper presents an analytical model for the resolution of the problem $\left(\mathcal{P}_{b}\right)$, avoiding the need of specific location information (such as GPS). We are only focused on the mathematical aspects in order to show that a resolution of the problem $\left(\mathcal{P}_{b}\right)$ is possible by using the LQI (the use of RSSI could be another alternative). From some topics presented in this paper, we have derived an energy efficient protocol for dcluster formation in WSN which is under evaluation and will be presented in a future publication.

\section{AnAlytical Model}

In this section, we develop an analytical model to derive an heuristic which resolves the problem $\left(\mathcal{P}_{b}\right)$ defined in the equation (4) under the constraints $\left(\mathcal{C}_{1}\right)$ and $\left(\mathcal{C}_{2}\right)$. Assume that nodes are randomly distributed in a field where the Base Station (BS) is located in the middle, i.e location $(B S)=(0,0)$. Since the main objective of most applications is to forward data packets towards the Base Station, we also assume that the BS forms the first cluster. Next clusters are then formed in a circular way around this first cluster.

\section{A. First Clusterhead Formation}

Initially, the Base Station (BS) forms the first cluster. Next clusters will be constructed around this first one. At this step, the set $\mathcal{S}$ of the known clusterheads is defined by:

$$
\mathcal{S}=\{b s\}
$$

The set $\Omega$ of clusterized nodes is defined by:

$$
\Omega=\mathcal{N}_{1}(b s)
$$

\section{B. Emissary Nodes Definition}

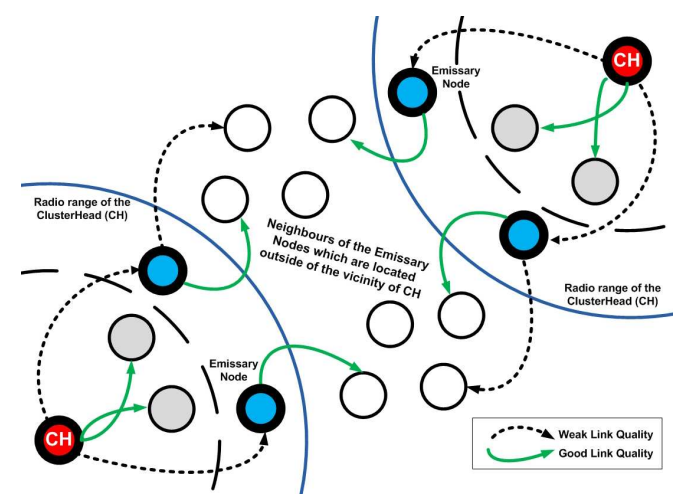

Fig. 2. Emissary Nodes determined by Link Quality
Respectively to each known cluster head in $x \in \mathcal{S}$ the "Emissary Nodes" of $x$ (Fig. 2) are the sensors which form weak links (small LQI values) with $x$, that is to say, the nodes located in the vicinity of the cluster head $x$ which forms with $x$ a link having a quality under a given $\ell_{\min }$ threshold. Each "emissary node" should also have at least one good link with another node which is located outside of the neighbourhood of $x$.

For each $x \in \mathcal{S}$, the set $\omega_{1}$ of the potential emissary nodes is defined by:

$$
\omega_{1}(x)=\left\{y \in \mathcal{N}_{1}(x) \backslash\{x\}, \quad 0<\ell(x, y) \leq \ell_{\text {min }}\right\}
$$

Let $x \in \mathcal{S}$, for each $y \in \omega_{1}(x)$, the set $\varpi_{1}(x, y)$ of nodes which are located outside of the neighbourhood of $x$ and which have a good link with $y$ is defined by:

$$
\varpi_{1}(x, y)=\left\{z \in V \backslash \mathcal{N}_{1}(x), \quad \ell(y, z)>\ell_{\max }\right\}
$$

Then, for each $x \in \mathcal{S}$, the set $\mathcal{W}_{1}$ of the emissary nodes is defined by:

$$
\mathcal{W}_{1}(x)=\left\{y \in \omega_{1}(x), \quad \varpi_{1}(x, y) \neq \emptyset\right\}
$$

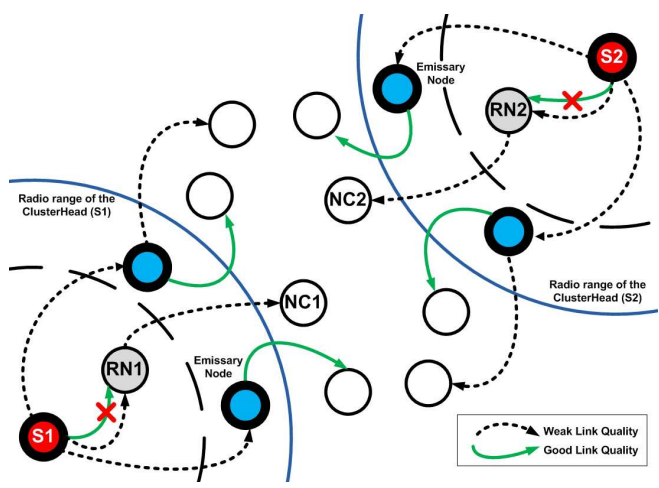

Fig. 3. Emissary Nodes determined by Link Quality

In equation (9), the condition $\varpi_{1}(x, y) \neq \emptyset$ is an important baseline of the analysis presented in this paper. So, to have a good idea of the remoteness of nodes in an environment which is subjected to some unreliabilities of the wireless links, this condition is necessary. For instance, if we consider the situation as shown in Fig. 3 where the link between the cluster head $S 1$ and the regular node $R N 1$ is not good (small LQI value, $\left.\ell(S 1, R N 1) \leq \ell_{\text {min }}\right)$ even if the two nodes are near to each other. Then under the condition $\varpi_{1}(x, y) \neq \emptyset, R N 1$ is not an emissary node of the cluster head $S 1$ because $R N 1$ does not have any good link with a node located outside of the 1-hop neighbourhood of $S 1$.

\section{Second Clusterhead Calculation}

The best emissary nodes $\left(\mathcal{B}_{E}\right)$ are used to form the relationship between clusters, in such a way that the subset $\mathcal{R}_{T}$, defined by the equation (10), builds a k-dominating set which is also $\mathrm{k}$-connected $(k=1)$. So at the end of the clustering 
process $\mathcal{R}_{T}$ forms a connected backbone of $\Omega$ in terms of data routing.

$$
\mathcal{B}_{E}=\cup_{i}\left\{b e_{i}\right\} \quad \text { and } \quad \mathcal{R}_{T}=\mathcal{S} \cup \mathcal{B}_{E}
$$

Then, the first best emissary node $b e_{1}$ is selected as the the emissary of $b s$ with which the BS forms a link of which the LQI is smaller. Let $b e_{1} \in \mathcal{W}_{1}(b s)$ defined by :

$$
\ell\left(b s, b e_{1}\right)=\min _{y \in \mathcal{W}_{1}(b s)} \ell(b s, y)
$$

We define $\Gamma\left(b s, b e_{1}\right)$ as the set of the emissaries of the first clusterhead, i.e $b s$, which are located in the neighbourhood of the best emissary node, i.e $b e_{1}$ :

$$
\Gamma\left(b s, b e_{1}\right)=\mathcal{W}_{1}(b s) \cap \mathcal{N}_{1}\left(b e_{1}\right)
$$

$\mathcal{H}\left(b e_{1}, b s\right)$ is defined as the set of the emissaries of the best emissary nodes which are located outside of the vicinity of the first clusterhead $(b s)$ :

$$
\mathcal{H}\left(b e_{1}, b s\right)=\mathcal{W}_{1}\left(b e_{1}\right) \backslash\left\{\mathcal{W}_{1}\left(b e_{1}\right) \cap \mathcal{N}_{1}(b s)\right\}
$$

For each $x \in \mathcal{H}\left(b e_{1}, b s\right), \lambda(x)$ is the best LQI value amongst the links formed by the node $x$ with each node $y \in \Gamma\left(b s, b e_{1}\right)$ :

$$
\lambda(x)=\max _{y \in \Gamma\left(b s, b e_{1}\right)} \ell(x, y)
$$
by:

The second clusterhead $c h_{2} \in \mathcal{H}\left(b e_{1}, b s\right)$ is then defined

$$
\lambda\left(c h_{2}\right)=\min _{x \in \mathcal{H}\left(b e_{1}, b s\right)} \lambda(x)
$$

At this step, the set $\mathcal{S}$ of cluterheads is updated by:

$$
\mathcal{S}=\mathcal{S} \oplus\left\{\operatorname{ch}_{2}\right\}
$$

The set $\Omega$ of clusterized nodes is updated by:

$$
\Omega=\Omega \oplus \mathcal{N}_{1}\left(c h_{2}\right)
$$

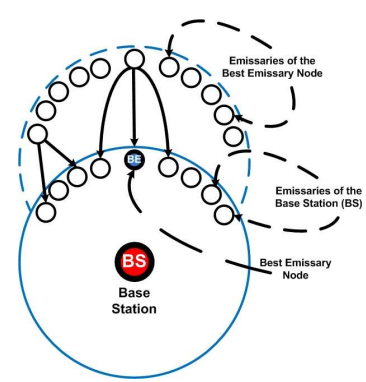

(a) Emissary Nodes

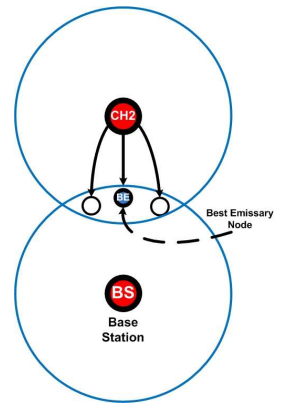

(b) Second Clusterhead
Fig. 4. The Second Clusterhead and The Best Emissary Node

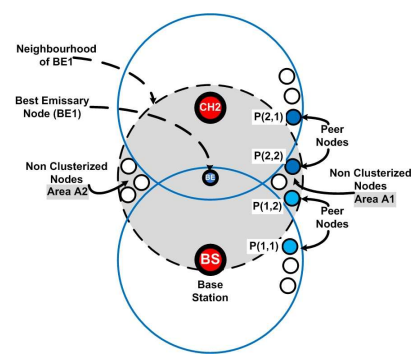

(a) $\mathcal{A}_{1} \cup \mathcal{A}_{2} \neq \emptyset$

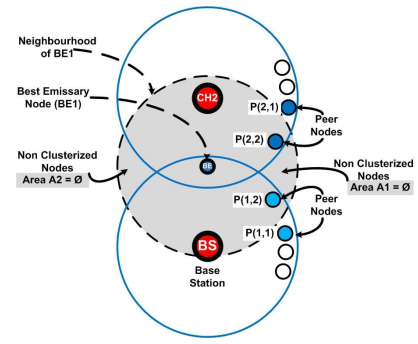

(b) $\mathcal{A}_{1} \cup \mathcal{A}_{2}=\emptyset$
Fig. 5. Peer Nodes in the third Clusterhead Calculation

\section{Third Clusterhead Calculation}

The non clusterized nodes located in the neighbourhood of $b e_{1}$ are divided in two areas $\mathcal{A}_{1}$ and $\mathcal{A}_{2}$ (Fig. 5(a)):

$$
\mathcal{A}_{1} \cup \mathcal{A}_{2}=\mathcal{N}_{1}\left(b e_{1}\right) \backslash\left\{\mathcal{N}_{1}\left(b e_{1}\right) \cap \Omega\right\}
$$

The third Clusterhead can be calculated relatively to $\mathcal{A}_{1}$ or $\mathcal{A}_{2}$. Choosing one of these two areas will determine the direction in which next clusters are to be formed around the first clusterhead (BS). If one of these two areas is empty, then choose the non empty area. Suppose that we choose the direction of the $\mathcal{A}_{i}$ area $(i=1$ or 2$)$.

$$
\begin{gathered}
\Gamma\left(b s, b e_{1}\right)=\mathcal{W}_{1}(b s) \cap \mathcal{N}_{1}\left(b e_{1}\right) \\
\mathcal{H}\left(b s, b e_{1}\right)=\mathcal{W}_{1}(b s) \backslash\left\{\mathcal{W}_{1}(b s) \cap \mathcal{N}_{1}\left(b e_{1}\right)\right\}
\end{gathered}
$$

Although we are in dense WSNs, $\mathcal{A}_{1} \cup \mathcal{A}_{2}$ could be an empty set. So, if $\mathcal{A}_{1} \cup \mathcal{A}_{2} \neq \emptyset$, then the peer nodes $\left(p_{1,1}, p_{1,2}\right)$ such that $p_{1,1} \in \mathcal{H}\left(b s, b e_{1}\right)$ and $p_{1,2} \in \mathcal{A}_{i}$ are defined by (Fig. $5(\mathrm{a}))$ :

$$
\ell\left(p_{1,1}, p_{1,2}\right)=\max _{\left(x \in \mathcal{H}\left(b s, b e_{1}\right), y \in \mathcal{A}_{i}\right)} \ell(x, y)
$$

else if $\mathcal{A}_{1} \cup \mathcal{A}_{2}=\emptyset$, then the peer nodes $\left(p_{1,1}, p_{1,2}\right)$ such that $p_{1,1} \in \mathcal{H}\left(b s, b e_{1}\right)$ and $p_{1,2} \in \Gamma\left(b s, b e_{1}\right)$ are defined by (Fig. $5(\mathrm{~b}))$ :

$$
\ell\left(p_{1,1}, p_{1,2}\right)=\max _{\left(x \in \mathcal{H}\left(b s, b e_{1}\right), y \in \Gamma\left(b s, b e_{1}\right)\right)} \ell(x, y)
$$

Likely, the peer nodes $\left(p_{2,1}, p_{2,2}\right)$ such that $p_{2,1} \in$ $\mathcal{H}\left(c h_{2}, b e_{1}\right)$ and $p_{2,2} \in \mathcal{A}_{i}$ or $p_{2,2} \in \Gamma\left(c h_{2}, b e_{1}\right)$ are defined by the equations (21) or (22). In the case of $\mathcal{A}_{1} \cup \mathcal{A}_{2}=\emptyset$, then $p_{2,2}$ must be also in $\mathcal{N}_{1}\left(p_{1,2}\right)$.

Let $\mathcal{P}$ the non empty set defined as follows:

if $\mathcal{N}_{1}\left(p_{1,1}\right) \cap \mathcal{N}_{1}\left(p_{2,1}\right) \neq \emptyset$

$$
\mathcal{P}=\mathcal{N}_{1}\left(p_{1,1}\right) \cap \mathcal{N}_{1}\left(p_{2,1}\right)
$$

else

$$
\mathcal{P}=\mathcal{N}_{1}\left(p_{1,2}\right) \cap \mathcal{N}_{1}\left(p_{2,2}\right)
$$

Finally, the third clusterhead $c h_{3} \in \mathcal{P}$ (Fig. 6) is then defined by:

$$
\ell\left(c h_{3}, p_{2,1}\right)=\min _{x \in \mathcal{P} \cap \mathcal{W}_{1}\left(p_{1,1}\right)} \ell\left(x, p_{2,1}\right)
$$




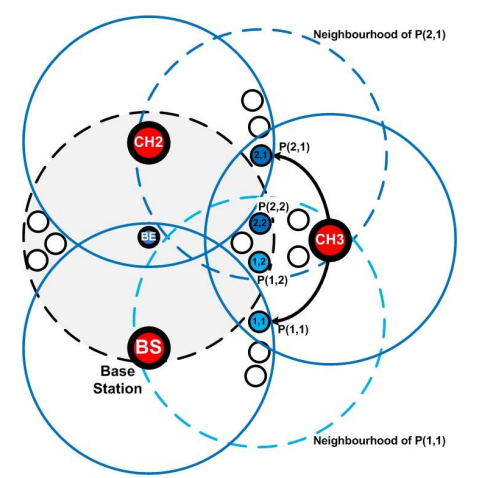

Fig. 6. Determination of the Next Clusterhead

\section{E. Avoiding Non Clusterized Nodes between Clusters}

As shown in Fig. 7, some non clusterized nodes $\left(x_{n c n}\right)$ might happen to appear between the formed clusters. For instance, if after the third cluster head selection $\left(c h_{3}\right)$, some nodes in the area $\mathcal{A}_{1}$ (Fig. 5(a)) are still non clusterized (Fig. $7)$, in this case, the current clusterhead should be cancelled. Otherwise, the area $\bar{\Omega}$ formed by the zone bounded by the peripheral nodes of $\Omega$ would not be convex because $x_{n c n} \notin \Omega$. That is to say that the constraint $\left(\mathcal{C}_{2}\right)$ would not be satisfied at the end of the clustering process.

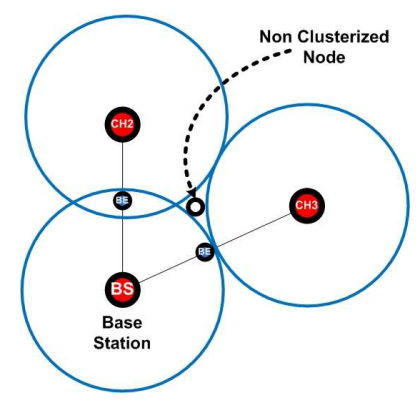

Fig. 7. Avoiding Non Clusterized Nodes between Clusters

Let $\hbar$ the node $\hbar \in \mathcal{P}$ (Fig. 6) such that:

$$
\ell\left(\hbar, p_{2,1}\right)=\min _{x \in \mathcal{P} \cap \mathcal{W}_{1}\left(p_{1,1}\right)} \ell\left(x, p_{2,1}\right)
$$

Let $\mathcal{X}$ the set defined by the following equation:

$$
\mathcal{X}=\mathcal{P} \cap \mathcal{W}_{1}\left(p_{1,1}\right) \backslash\{\hbar\}
$$

The node $\hbar$ is excluded from the clusterhead selection process, and the next clusterhead $c h_{3} \in \mathcal{X}$ is then defined by :

$$
\ell\left(\operatorname{ch}_{3}, p_{2,1}\right)=\min _{(x \in \mathcal{X})} \ell\left(x, p_{2,1}\right)
$$

This process is repeated until all nodes in the area $\mathcal{A}_{1}$ are clusterized.

At this step, the set $\mathcal{S}$ of cluterheads is updated by:

$$
\mathcal{S}=\mathcal{S} \oplus\left\{c h_{3}\right\}
$$

The set $\Omega$ of clusterized nodes is then updated by:

$$
\Omega=\Omega \oplus \mathcal{N}_{1}\left(c h_{3}\right)
$$

\section{F. Best Emissary Node (BE) Calculation}

Once the third cluster head is known, the second best emissary node $\left(b e_{2}\right)$ between the BS and $c h_{3}$ is then selected as the emissary node of the BS with which $c h_{3}$ forms a link of which the LQI is smaller (Fig. 8).

$$
\Gamma\left(b s, c h_{3}\right)=\mathcal{W}_{1}(b s) \cap \mathcal{N}_{1}\left(c h_{3}\right)
$$

Let $b e_{2} \in \Gamma\left(b s, c h_{3}\right)$ the node defined by (Fig. 8):

$$
\ell\left(c h_{3}, b e_{2}\right)=\min _{x \in \Gamma\left(b s, c h_{3}\right)} \ell\left(\operatorname{ch}_{3}, x\right)
$$

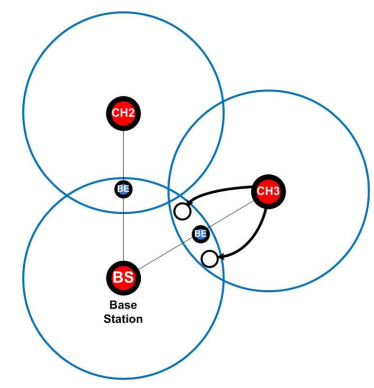

Fig. 8. Adjusting The Best Emissary Node (BE)

\section{Global Heuristic}

The proposed heuristic is presented in table I below. Assume that the nodes are randomly distributed in a field where the Base Station (BS) is located in the middle, i.e location $(B S)=$ $(0,0)$. The Base Station initially forms the first cluster which is also the reference cluster around which next clusters are to be constructed as in sections V.C, V.D, V.E and V.F. When the intersection between the current cluster under construction and the cluster that follows the reference cluster is not empty, then this latter will play the role of the reference cluster. The area is clusterized in this manner until the stop condition is met. Here we stop clustering at the end of the first round around the Base Station (Fig. 9).

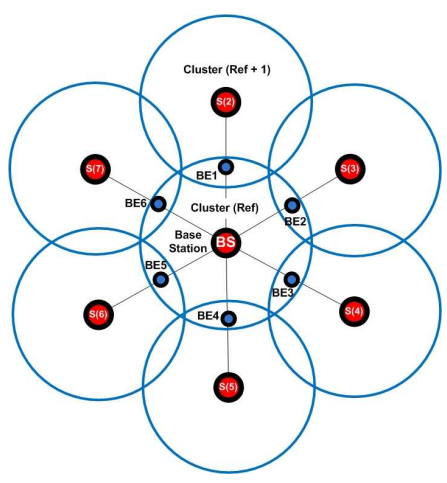

Fig. 9. Cluster Formation Around the First Clusterhead 
TABLE I

GLOBAL HeURISTIC

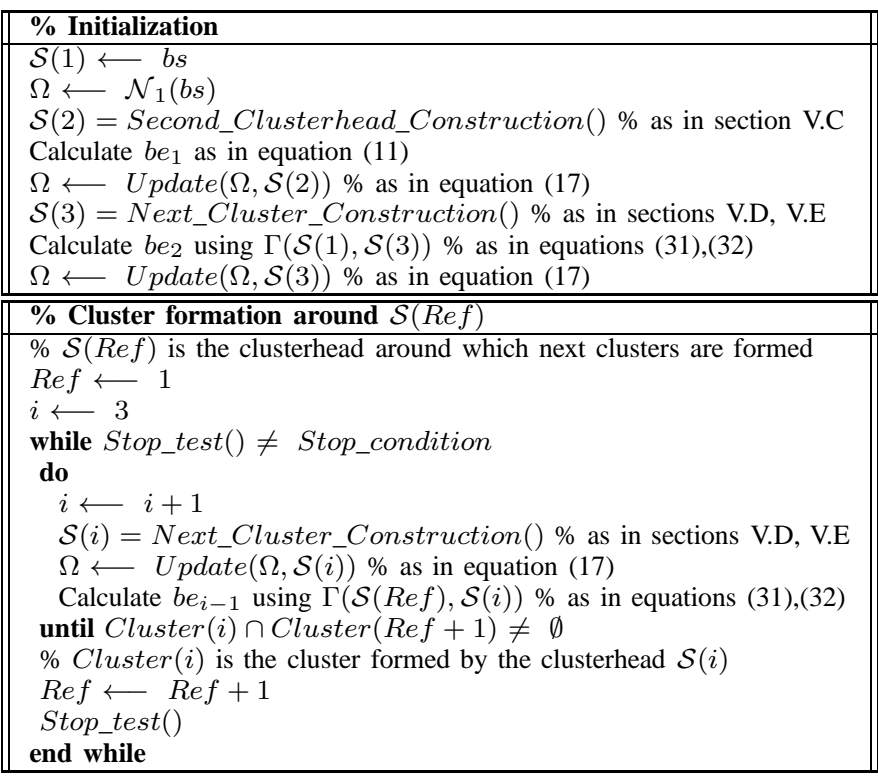

\section{LQI MOdEL FOR SimUlation PURPoSES}

The LQI values are calculated by using the $\ell(x, y)$ function defined below:

$$
\begin{gathered}
\ell(x, y)=\alpha+\frac{\beta * \log \left(1+\left(\gamma(x, y)-\gamma_{\min }(x)\right)\right)}{\log \left(1+\gamma_{\max }(x)\right)} \\
\left.\gamma_{(} x, y\right)=\frac{1}{d(x, y)} \\
\gamma_{\min }(x)=\min _{y \in \mathcal{N}_{1}(x)} \gamma(x, y) \\
\gamma_{\max }(x)=\max _{y \in \mathcal{N}_{1}(x)} \gamma(x, y)
\end{gathered}
$$

where $\alpha=50, \beta=255$ and $d(x, y)$ is the distance separating $y$ from $x$.

The choice of this model is guided by experimental results shown in [12] and [13] which stated that the LQI decreases when the distance between nodes increases in Zigbee-based WSN. As we can see, $\ell(x, y) \neq \ell(y, x)$. Hence, the model allows to take into account asymetrical aspects of wireless links.

For moteiv's Tmote Sky [15] sensors equipped with chipcon's CC2420 [16], the LQI values range from 50 to 110 . Even so, we stick with the ZigBee standard [1],[2] because some manufacturers, such as Sun-SPOT [17] and WiEye [18], are still using the standard LQI values. Then, we use the standard values (i.e. [0,255]), instead of those of CC2420.

This LQI model is only used for simulation purpose, so sensor nodes do not compute these above formulas.

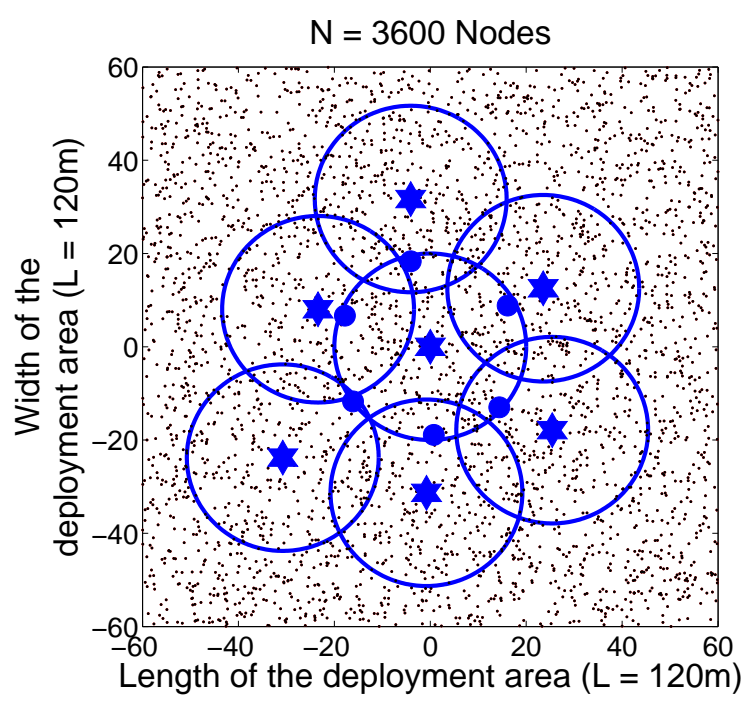

Fig. 10. Uniform Random Deployment: The average clusterhead locations (stars) with its respective clusters (rings) and the average best emissary node locations which are represented by the small circle on the outskirts of the first cluster (BS).

\section{Simulation RESUlTS}

In the simulation model $\mathrm{N}$ nodes are randomly (according to a uniform distribution) deployed over a square area $[-L,+L]$ of length $L=60 \mathrm{~m}$. The BS is located at the $(0,0)$ position. All nodes, including the BS, have the same transmission range $(R=20 m)$.

Simulations are run for a network size ranging from 2800 to 7200 nodes. The results presented here are obtained by averaging the results for 10 different simulations for each scenario. For each simulation, a new random node layout is used. In all simulation results presented below, $\ell_{\max }=230$ and $\ell_{\min }=70$.

The figures 10 and 11 show the average clusterhead and best emissary node locations.

These results show that the heuristic works as expected because the similarity of the simulation results (Fig. 10,11) compared with the expected theoretical result as shown in Fig. 9 is coherent.

For both the grid and the random deployment topologies, the figure 12 plots the average distance between the two closest caryommes when the node density is increasing. This result shows that the minimum distance separating the clusterheads increases as the node density is increasing. This means that our analytical model is more accurate for dense networks and also for the uniform random deployment topology. This result is also expected because to have a similar result as shown in the figure 9, one should consider a network of which the density nodes tends to infinity.

The average distance between the two closest caryommes is smaller in the grid topology (Fig. 12). Because of the symmetry related to the nature of the deployment, a given node has some neighbours with which it forms links having the same quality. Consequently, the nodes on which the 


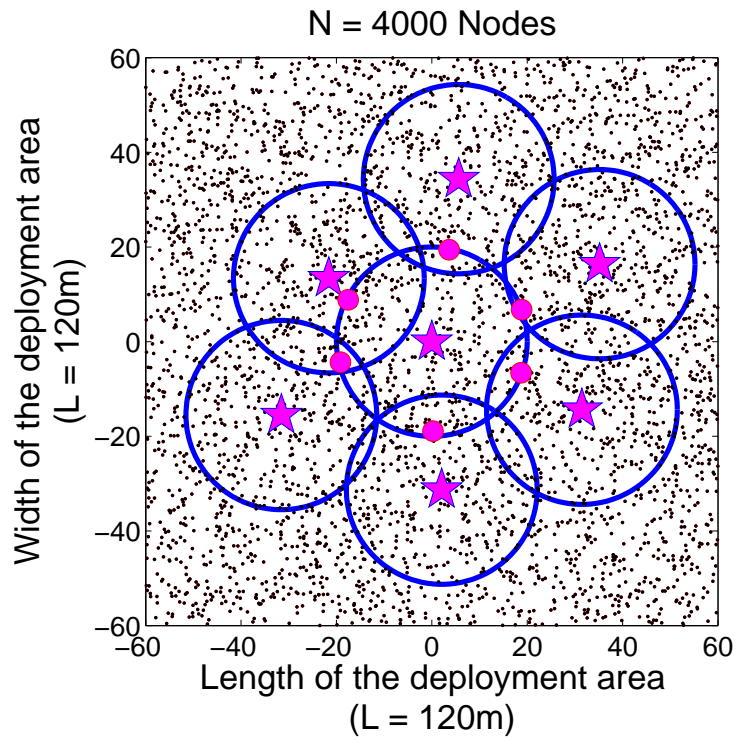

Fig. 11. Uniform Random Deployment: The average clusterhead locations (stars) with its respective clusters (rings) and the average best emissary node locations which are represented by the small circle on the outskirts of the first cluster (BS).

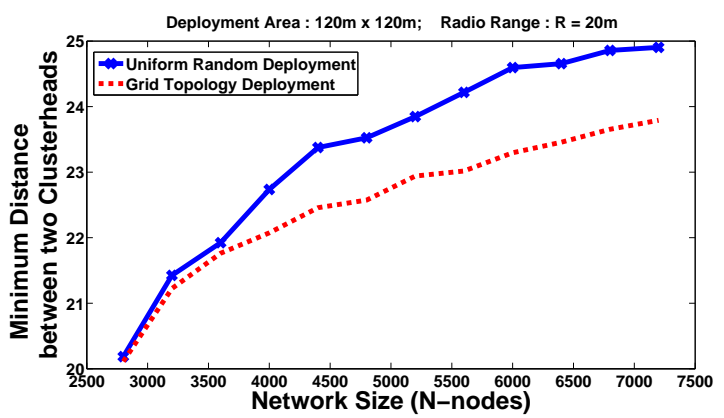

Fig. 12. Grid Topology vs Uniform Random Deployment: Average distance between the two closest caryommes at the end of the clustering process.

calculation of caryommes and emissaries rely on, are determined by its identifiers according to the total ordering in $V$ as defined by the equation (2). Then, compared to a grid deployment topology, the heuristic is more suitable to a WSN of which nodes are randomly distributed in a field.

\section{CONCLUSion AND Future Works}

In this paper, we have presented an analytical model based on the use of the LQI in order to derive an heuristic which forms an optimally one-dominating set in which the distance separating the two closest cluster heads is improved. During the clustering process, each caryomme selects in its 1-hop neighbourhood some nodes which are its emissaries. The relationship between two adjacent clusters is then created by the best emissary node located between the two respective cluster heads. This model constructs a k-dominating sub-sets of the wireless sensor network where each node within a cluster is at most $\mathrm{k}$ wireless hops away from its cluster head $(k=1)$, and also forms a connected backbone composed of the set of cluster heads plus the set of the best emissary nodes. Simulation results show that the proposed heuristic works as expected with respect to the expected theoretical results. Future works would focus on deriving an energy efficient protocol from all or some topics presented in this paper. To achieve this goal, one should bear in mind the specific constraints of sensors such as limited computational and memory capacities.

\section{REFERENCES}

[1] IEEE Std 802.15.4-2006. Wireless medium access control (mac) and physical layer (phy) specifications for low-rate wireless personal area networks (wpans). In IEEE Computer Society, 2006.

[2] Zigbee Alliance. Zigbee specification v1. Jun. 2005.

[3] C. Gao and R. Jantti. Link-state clustering based on ieee 802.15.4 mac for wireless ad-hoc/sensor networks. In Proc. IEEE Wireless Communications and Networking Conference, WCNC 2006, Volume 1, Page(s):499 - 504, Las Vegas, NV USA, Apr. 2006.

[4] W. B. Heinzelman, A. Chandrakasan, and H. Balakrishnan. An application-specific protocol architecture for wireless microsensor networks. IEEE Transactions on Wireless Communications, Vol. 1(4), pages 660-670, Oct. 2002.

[5] S. Fazackerley, A. Paeth, and R. Lawrence. Cluster head selection using rf signal strength. In Proc. of Canadian conference on electrical and computer engineering, CCECE, Newfoundland, Canada, May 2009.

[6] A.D. Amis, R. Prakash, T.H.P Vuong, and D.T. Huynh. Max-min dcluster formation in wireless ad hoc networks. In 9th Annual Joint Conference of the IEEE Computer and Communications Societies, 2000.

[7] F. G. Nocetti, J. S. Gonzalez, and I. Stojmenovic. Connectivity based k-hop clustering in wireless networks. Telecommunication Systems, Vol. 22, Numbers 1-4, pages 205-220, Jan. 2003.

[8] M.R. Brust, H. Frey, and S. Rothkugel. Dynamic multi-hop clustering for mobile hybrid wireless networks. In ACM Proc. of the 2nd international Conference on Ubiquitous information Management and Communication, ICUIMC'08, Vol. 01, Suwon, Korea, Jan. 2008.

[9] Y. Liu, J. Gao, L. Zhu, and Y. Zhang. A clustering algorithm based on communication facility in wsn. In WRI International Conference on Communications and Mobile Computing, CMC '09, Vol. 2, pages 76-80, Jan. 2009.

[10] F. Dai and J. Wu. On constructing k-connected k-dominating set in wireless networks. In Proc. of the 19th IEEE international Parallel and Distributed Processing Symposium, IPDPS'05, Vol. 01, Washington, DC, USA, Apr. 2005.

[11] K. Shin, A. Abraham, and Y. H. Sang. Self organizing sensor networks using intelligent clustering. In Proc. of International Conference on Computational Science and its Applications, vol. 3984, pp. 40-49, Glasgow, UK, May. 2006.

[12] J. Blumenthal, R. Grossmann, F. Golatowski, and D. Timmermann. Weighted centroid localization in zigbee-based sensor networks. In IEEE International Symposium on Intelligent Signal Processing, WISP, 2007.

[13] M. Becker, A.L Beylot, R. Dhaou, A. Gupta, R. Kacimi, and M. Marot. Experimental study: Link quality and deployment issues in wireless sensor networks. In Proc. NETWORKING 2009, LNCS 5550, pages 14-25, NETWORKING, Aachen, Germany, 2009.

[14] C. Diallo, M. Marot, and M. Becker. Single-node cluster reduction in wsn and energy-efficiency during cluster formation. In Proc. of the 9th IFIP Annual Mediterranean Ad Hoc Networking Conference, Med-HocNet 2010, IEEE Communications Society, Juan-Les-Pins, France, June. 2010.

[15] Tmote Sky. http://www.snm.ethz.ch/projects/tmotesky. Last access, Mar. 2010.

[16] CC2420 Radio. http://www.chipcon.com. Last access, Mar. 2010.

[17] Sun SPOT World. http://www.sunspotworld.com. Last access, Mar. 2010.

[18] EasySen WiEye Sensor Board. http://www.easysen.com/wieye.htm. Last access, Mar. 2010. 